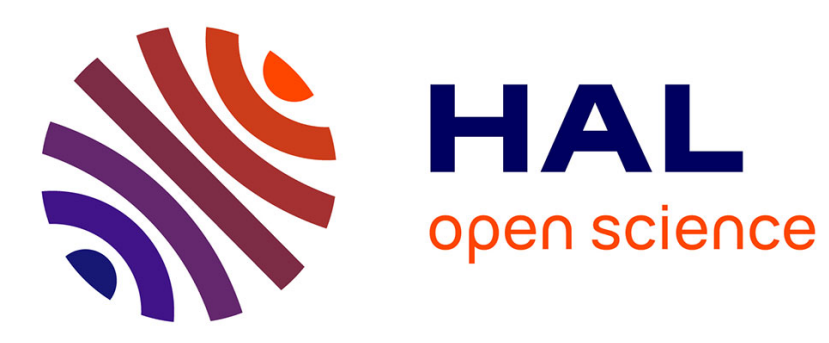

\title{
Power Oscillations Damping Controller for HVDC Inserted in Meshed AC Grids
}

Yankai Xing, Bogdan Marinescu, Mohamed Belhocine, Florent Xavier

\section{To cite this version:}

Yankai Xing, Bogdan Marinescu, Mohamed Belhocine, Florent Xavier. Power Oscillations Damping Controller for HVDC Inserted in Meshed AC Grids. 2018 IEEE PES Innovative Smart Grid Technologies Conference Europe (ISGT-Europe), Oct 2018, Sarajevo, Bosnia and Herzegovina. 10.1109/ISGTEurope.2018.8571732 . hal-02512398

\section{HAL Id: hal-02512398 https://hal.science/hal-02512398}

Submitted on 19 Mar 2020

HAL is a multi-disciplinary open access archive for the deposit and dissemination of scientific research documents, whether they are published or not. The documents may come from teaching and research institutions in France or abroad, or from public or private research centers.
L'archive ouverte pluridisciplinaire HAL, est destinée au dépôt et à la diffusion de documents scientifiques de niveau recherche, publiés ou non, émanant des établissements d'enseignement et de recherche français ou étrangers, des laboratoires publics ou privés. 


\title{
Power Oscillations Damping Controller for HVDC Inserted in Meshed AC Grids
}

\author{
Yankai Xing*, Bogdan Marinescu*, Mohamed Belhocine*, Florent Xavier ${ }^{\dagger}$ \\ ${ }^{*}$ Ecole Centrale de Nantes, LS2N-CNRS \\ 1, rue de la Noë, Nantes 44300, France \\ Email: \{Yankai.Xing, Bogdan.Marinescu\}@ec-nantes.fr \\ ${ }^{\dagger}$ RTE R\&D Division, Versailles, France \\ Email: florent.xavier@ rte-france.com
}

\begin{abstract}
This paper addresses the problem of damping interarea oscillations via power modulation of a VSC-HVDC link integrated in a power AC grid. Motivated by the fact that interarea modes may be at higher frequencies, close to other modes of the system, and classic tuning methods of standard (IEEE) power oscillations damping controller structures may not give satisfactory results. A reduced order model of a meshed AC grid with a HVDC link is proposed for control design. Based on this model which carefully integrates the dynamics of interest, a robust controller for the HVDC link is designed to damp interarea oscillations and enhance the damping of the other modes. Investigations with both linearized and nonlinear model of the system are carried out to settle and validate the approach. The efficiency and robustness of the proposed controller are tested and compared with standard controller structures.
\end{abstract}

Index Terms-Power system oscillations, HVDC, damping controller, inter-area modes, robustness

\section{INTRODUCTION}

With the growing scale of power grids, electromechanical oscillations problem has become an important aspect of power system stability. Nowadays, High Voltage Direct Current (HVDC) transmission systems have been gradually integrated in power grids. VSC (Voltage Source Converters)-HVDC transmission systems have the advantage of independent and fast control of active/reactive power. This plays an important role to maintain system stability and achieve a better damping of inter-area oscillation modes by the means of well designed supplementary POD (Power Oscillations Damping) controller in control loops [1].

In the past, the inter-area modes which were analyzed and controlled were the ones at low frequencies, around $0.2 \mathrm{~Hz}$ in Europe. These are the most spread modes of the transmission grid, i.e., the ones which involve the largest number of generators. For a HVDC inserted in a meshed AC grid as it is the case for the recent interconnection reinforcements in Europe (like, e.g., France-Spain and France-Italy links), interarea modes which are controllable with the HVDC converters may be at higher frequency, around $1 \mathrm{~Hz}$ (i.e., may concern a limited number of generators in the neighbor zone of the HVDC). In this frequency range, there are also other dynamics of the grid. The inter-area modes are thus close to other modes of different natures (see [2] and [3]) that may be disturbed by the POD controller of the HVDC. Using a classic IEEE
POD controller structure and tuning methodologies (see, e.g., [4]) may lead to ineffective damping for the inter-area modes and may destabilize the other ones. Hence, the objective of this paper is to provide a POD controller which improves the damping of this mode without deteriorating the damping of the others.

Advanced control (robust $H_{\infty}, H_{2}$, LQG or LMI pole placement) has already been proposed to improve performances and robustness of grid connected power electronics as, for example, in the research studies [5]-[9]. This kind of control usually needs a state representation of the system [10]. This is easy to provide when small systems are considered, but it becomes a difficult task for large grids, especially when interarea modes are the target. Indeed, in this case, a large-scale representation of the grid should be considered. This does not allow the direct use of standard reduction methods like based on balanced realisation state truncation [8] or Schur balanced model reduction [6] [7]. In [11], a methodology to capture the grid dynamics into a reduced order control model in the whole frequency range around the frequencies of the interarea modes to be damped is proposed. The same methodology is adopted in this paper but for the context of oscillations damping by VSC-HVDC power modulation. This is a way to simultaneously detect and consider the modes to be damped (as a target of the POD controller) and the other modes of grid which may be affected by the POD controller. Based on this model, the POD is here synthesized in the Linear Quadratic Gaussian (LQG) robust control framework in order to achieve coordination between the damping actions of several modes, inter-area and their neighbors in the frequency range. A comparative study is carried out to highlight difference between the investigated control approaches.

The paper is organised as follows: in Section II the modeling is given and the control problem is formulated. The basics of the control model and the new control framework are given in Section III. Its application is discussed in Section IV. Section $\mathrm{V}$ is devoted to concluding and perspectives of this work.

\section{PROBLEM FORMULATION}

\section{A. HVDC in a meshed AC grid}

Consider a HVDC transmission line inserted into a AC grid as shown in Fig. 1. The two converters are controlled by first- 
level regulation loops to fulfill local objectives. The POD is a second-level regulator devoted to grid objectives (damping of inter-area modes). The situation in Fig. 1 becomes particular

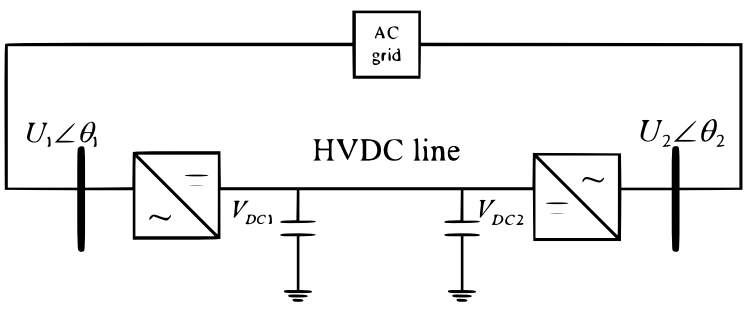

Fig. 1. HVDC inserted in an AC grid.

when the AC grid is highly meshed. As explained in Section $\mathrm{I}$, in this case, the inter-area modes which can be damped with the HVDC are at higher frequencies and close to the other modes of the AC system. The plant to be controlled by the POD regulator $(H(s)$ in Fig. 2) is much more difficult to be defined/extracted from the combined HVDC and AC grid model which is a large-scale model. A methodology to extract the dynamics of interest into a small-scale control model is represented in Section III-A.

This situation is often encountered in grids like the European one. A benchmark with these particularities is presented in the next section.

\section{B. Test system}

In this paper, a power system with 19 generators and one HVDC line is analysed. All generators are equipped with AVR (Automatic Voltage Regulator) and PSS (Power System Stabilizer). The full nonlinear model of this system, which is of order 724, is linearized around an equilibrium point with Eurostag software [12] to obtain the full linear model of the same order.

The modes which can be damped via HVDC control are the ones highly observable and controllable, i.e., which have high residues in the open-loop transfer $H(s)$ as shown in Fig. 2 [13], [14]. Table I provides the highest residues modes found in the full linear model. For each mode (characterized by its damping and frequency), most participating machines are reported in the table and categorized into two sets (' + ' and '-') of generators oscillating against each other. For example, the most participating generator to mode 2 is GE_911 and the second one is GE_917. These two machines are swinging against each other. This is concluded from the difference of the phase of right eigenvector components of the mode which corresponds to the machine angles, which is close to $180^{\circ}$. This mode is an inter-area mode as it involves two coherent groups of generators swinging against each other at a low frequency [15].

From Table I, one can notice that mode 2 has a poor damping (4.5\%) and the operation of the system in the presence of a lightly damped inter-area mode is a serious issue and has to be considered in the control design phase. Besides, notice that this inter-area mode is at $0.88 \mathrm{~Hz}$ and several modes (highly damped (6 to 10 ) or poorly damped (1 to 5 )) are at frequencies close to this one.

It should be highlighted that mode 8 is not an inter-area mode since related generators (GE_921, GE_922) swinging against each other are in the same area and connected to the same node. It is so-called local mode.

\section{Classic POD and its limitations}

Inter-area modes can be damped with additional high-level POD control loops for the converter as mentioned in Section I. The terminal difference of angles, $\Delta \theta=\theta_{1}-\theta_{2}$, is usually used as an additional control signal to provide power (active power $P$ and/or reactive power $Q$ ) modulation to the aforementioned first-level loops as illustrated in Fig. 2 [14]. In this paper, the reactive power modulation $Q$ is considered as a first investigation.

The classic IEEE HVDC POD controller [4] has the same structure as the PSS (Power System Stabilizer) for generators, which contains a gain $K$, a wash-out filter and $n$ phase leadlag blocks (Fig. 2). It is defined by the transfer function given by (1).

$$
H_{P O D}(s)=K\left(\frac{T_{w} s}{1+T_{w} s}\right)\left(\frac{1+T_{1} s}{1+T_{2} s}\right)^{n}
$$

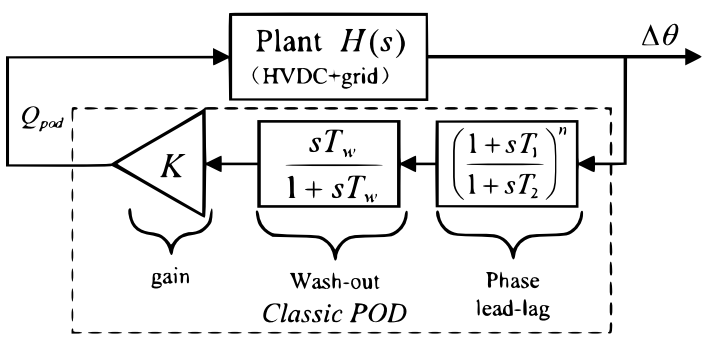

Fig. 2. Structure of classic POD.

The washout filter is usually used in practice as an interface between the measured signal and the used one in the control loop in order to wash-out (reject) the steady-state component of the measures, while passing transient ones [16]. The classic tuning of parametric technique consists of two steps (see, e.g. [13]) : i) Compute $T_{1}$ and $T_{2}$ in order to compensate the phase of the residue of the mode to $180^{\circ}$, ii) Adjust the gain $K$ to obtain the desired damping.

This methodology can be precisely implemented for one mode and it can be approximatively extended to multiple modes. However, only the residue of the mode to be damped in the plant is used as information from the grid. And in a situation like the one presented above, interactions with the modes having close frequencies may lead to poor results. Indeed, this is put into evidence on this test system when a target damping of $10 \%$ was fixed for mode 2 . The tuning methodology mentioned above yields: $K=2.5614$, $T_{1}=0.4746, T_{2}=0.0688, T_{w}=1$ and $n=2$. However, using this tuning approach, the real damping of this mode is only about $6 \%$ as reported in Table II and Fig. 6 which 
TABLE I

MODES OF LINEARIZATION

\begin{tabular}{|c|c|c|c|c|c|c|c|}
\hline \multirow{2}{*}{ No. } & \multirow{2}{*}{ Mode } & \multirow{2}{*}{$\begin{array}{l}\text { Damping } \\
\xi(\%)\end{array}$} & \multirow{2}{*}{$\begin{array}{l}\text { Freq. } \\
(\mathrm{Hz})\end{array}$} & \multicolumn{2}{|c|}{ Mode shape (participation mag (\%)) } & \multicolumn{2}{|c|}{ Residue } \\
\hline & & & & + & - & ABS MAG & Phase \\
\hline 1 & $-1.62+\mathrm{j} 8.19$ & 19.5 & 1.30 & GE_914 (100) & GE_913 (32.4) & 0.0157 & 35.0 \\
\hline 2 & $-0.24+j 5.53$ & 4.5 & 0.88 & GE_911 (100) & GE_917 (68.8) & 0.0181 & 83.4 \\
\hline 3 & $-0.53+\mathrm{j} 5.29$ & 10.1 & 0.84 & GE_917 (100) & GE_918 (55.1) & 0.0129 & -56.2 \\
\hline 4 & $-0.40+j 4.79$ & 8.3 & 0.76 & GE_918 (44.3) & GE_912 (100) & 0.0038 & -33.3 \\
\hline 5 & $-0.33+\mathrm{j} 3.29$ & 10.1 & 0.52 & GE_915 (100) & GE_918 (17.7) & 0.0121 & 104.5 \\
\hline 6 & $-18.83+\mathrm{j} 7.21$ & 93.3 & 1.14 & GE_921, GE_922 (100) & GE_923, GE_924 (74.1) & 0.0034 & 14.5 \\
\hline 7 & & 22.9 & 1.04 & GE_914 (100) & GE_911 (68.3) & 0.0125 & 151.5 \\
\hline 8 & -19.32 & 94.8 & 1.03 & GE_921 (100) & GE_922 (37.6) & 0.0117 & 118.9 \\
\hline 9 & $-20.33+j 4.86$ & 97.2 & 0.77 & GE_921, GE_922 (84.5) & GE_927 (100) & 0.0026 & -168.1 \\
\hline 10 & $-18.72+j 3.35$ & 98.4 & 0.53 & GE_913 (33.4) & GE_912 (100) & 0.0072 & 136.1 \\
\hline
\end{tabular}

is much below the tuning target of $10 \%$. It should be noted that the damping of mode 1 is improved, even this is not the target of the designed POD controller. This is due to the fact that phase compensator of the POD compensates the residue phase of mode 1 close to $180^{\circ}$. Despite the fact that this can be considered as an advantage, it may act also in the opposite way as the case of mode 4 .

To overcome this difficulty, a state-space method which takes into account all involved dynamics (interacting modes at a specific range of frequencies) is used as explained in next section.

\section{NEW CONTROL FRAMEWORK}

\section{A. Control model}

In the situation mentioned in Section II, a dynamic control model is necessary to capture information for the AC grid. All this information exists in a state-representation systematically used by all the advanced control methods. In [11], a reduced order model has been proposed by an identification approach based on the frequency responses. The same approach will be adopted in our context to obtain a suitable control model.

Starting from a list of target modes to be damped $\Lambda=$ $\left\{\lambda_{1}, \ldots, \lambda_{l}\right\}$, this method provides a reduced order transfer function $H(s)$ in the form given by (2). It approximates the open-loop transfer function $H(s)$ in Fig. 2 in a specific range of frequencies containing different modes of $(\Lambda)$.

$$
\begin{aligned}
\tilde{H}(s) & =\frac{A(s)}{B(s)}+\frac{P(s)}{Q(s)} \\
\frac{A(s)}{B(s)} & =\sum_{k=1}^{l}\left[\frac{r_{k}}{s-\lambda_{k}}+\frac{\bar{r}_{k}}{s-\bar{\lambda}_{k}}\right],
\end{aligned}
$$

where $r_{k}$ is the residue of mode $\lambda_{k}$. Polynomials $P(s)$ and $Q(s)$ are chosen of the following form:

$$
\begin{aligned}
& P(s)=p_{n-1} s^{n-1}+\ldots+p_{0} \\
& Q(s)=s^{n}+q^{n-1} s^{n-1}+\ldots+q_{0}
\end{aligned}
$$

and their coefficients are computed to minimize objective function (5) such that the reduced-order model fits the full model system in the working frequency band.

$$
\begin{aligned}
& J= \sum_{\omega_{\Lambda}^{-} \leq \omega_{k} \leq \omega_{\Lambda}^{+}}\left[\alpha_{k}\left(A_{k}-\left|\tilde{H}\left(i \omega_{k}\right)\right|\right)^{2}+\right. \\
&\left.\beta_{k}\left(\varphi_{k}-\arctan \left(\tilde{H}\left(i \omega_{k}\right)\right)\right)^{2}\right]
\end{aligned}
$$

where $\left(A_{k}, \omega_{k}\right)$ and $\left(\varphi_{k}, \omega_{k}\right)$ are points of the Bode plots of the transfer of the full model $H(s), \omega_{\Lambda}^{-}$and $\omega_{\Lambda}^{+}$define the frequency working band (from $\Lambda$ ). The weights $\alpha_{k}, \beta_{k}$ are used to manage the trade-off between magnitude and phase fitting and, eventually, to give priority to the fitting at specific frequencies. It should be noted that the degree $n$ of polynomials $(P(s)$ and $Q(s))$ is chosen by trial and error starting from low values and increasing it until a sufficient degree of precision is reached for the curves fitting.

For the example considered here, $\Lambda$ is composed by the first 5 modes in Table I, $\omega_{\Lambda}^{-}=1.256 \mathrm{rad} / \mathrm{s}$ and $\omega_{\Lambda}^{+}=13.8 \mathrm{rad} / \mathrm{s}$, $\alpha_{k}=1, \beta_{k}=1$. The obtained results are shown in Fig. 3 where the reduced model fits the full model in the considered frequencies range $\left[\omega_{\Lambda}^{-}, \omega_{\Lambda}^{+}\right]$.

\section{B. LQG Background}

One of the simplest robust controllers is the Linear Quadratic Gaussian control (see, e.g., [10], [17]). It uses the following state-space representation of the system:

$$
\begin{aligned}
& \dot{x}=A x+B u+w_{d} \\
& y=C x+D u+w_{n}
\end{aligned}
$$

which can be easily obtained from $\tilde{H}(s) . w_{d}$ and $w_{n}$ are, respectively, state and measurement noises with covariance matrices $W$ and $V$. The solution of the LQG control problem is a control law $u(t)$ which stabilizes the closed-loop and minimizes the quadratic performance index:

$$
J_{L Q G}=E\left\{\lim _{T \rightarrow \infty} \frac{1}{T} \int_{0}^{T}\left[x^{T} Q x+u^{T} R u\right] d t\right\}
$$

where $E\{\cdot\}$ is the expectation operator, $Q$ and $R$ are weighting matrices, which are used as design parameters, along with $W$ and $V$, for the synthesis of the controller gains $K_{r}$ and $L$ :

$$
\begin{gathered}
K_{r}=R^{-1} B^{T} X \\
L=Y C^{T} V^{-1}
\end{gathered}
$$



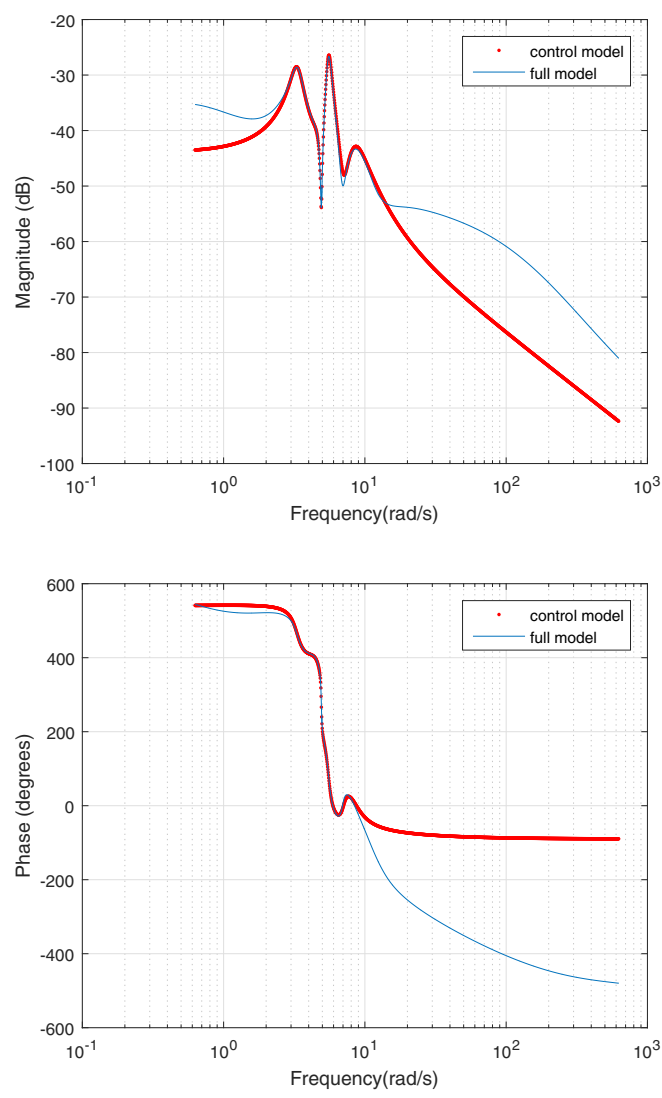

Fig. 3. Curve fitting of Bode plots of the full model and control model.

where $X$ and $Y$ are solutions of the following two Riccati equations:

$$
\begin{aligned}
& X A+A^{T} X-X B X^{-1} B^{T} X+Q=0 \\
& Y A^{T}+A Y-Y C^{T} R^{-1} C Y+W=0 .
\end{aligned}
$$

Notice that (8) is the solution of the control problem (a state feedback), while (9) gives the gain $L$ of the Kalman filter (used for state estimation):

$$
\dot{\hat{x}}=A \hat{x}+B u+L(y-C \hat{x}) .
$$

Hence, the structure of the LQG controller is given by $u=$ $-K_{r} \hat{x}$ where $\hat{x}$ is provided by the above Kalman filter. Fig. 4 illustrates a general representation of a $\mathrm{LQG}$ regulator.

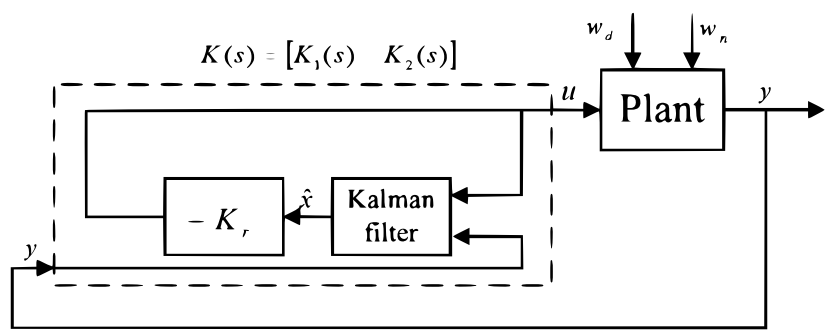

Fig. 4. General scheme of LQG controller.

\section{Application to the test CASE}

\section{A. Controller design}

In the previous section, the LQG control scheme for the stabilization of systems in the form (6) was presented. In our case, the difference of angles $\Delta \theta$ is considered as the output of the plant representing the grid with the HVDC link. The same wash-out filter used in Fig. 2 is added in LQG POD controller. With this in mind, considering the new output $\Delta \theta_{w}$, which corresponds to the output of the wash-out filter as depicted in Fig. 5, the LQG controller is designed based on an equivalent model $H^{\prime}(s)$, which includes the reduced model $(H(s))$ in series with the wash-out filter with $T_{w}=1$.

Usually, the weighting matrices $Q$ and $R$ are diagonal matrices and their entries are chosen according to state dynamics. A higher value in $Q$ (or, equivalently, a low value in $R$ ) will lead to faster dynamics (more control action on that specific state), i.e., more performances. A higher value in $R$ will conversely lead to a lower control level in that state direction, i.e., to more robustness. The same holds for the choice of $V$ and $W$ for the estimator synthesis. This control framework provides thus an easy way to handle the trade-off performances/robustness. The numerical values used for case treated here are $Q=I$, $R=10^{-2} \times I ; W=I, V=10^{-4} \times I$. The LQG control is implemented as in Fig. 5 and the transfer matrix of regulator is:

$$
Q_{p o d}=-K_{r} \hat{x}=-K_{r}(s I-A+L C)^{-1}\left(\begin{array}{ll}
B & L
\end{array}\right)\left(\begin{array}{c}
u \\
\Delta \theta_{w}
\end{array}\right)
$$

which $K_{r}$ and $L$ are obtained by Matlab function lqr.

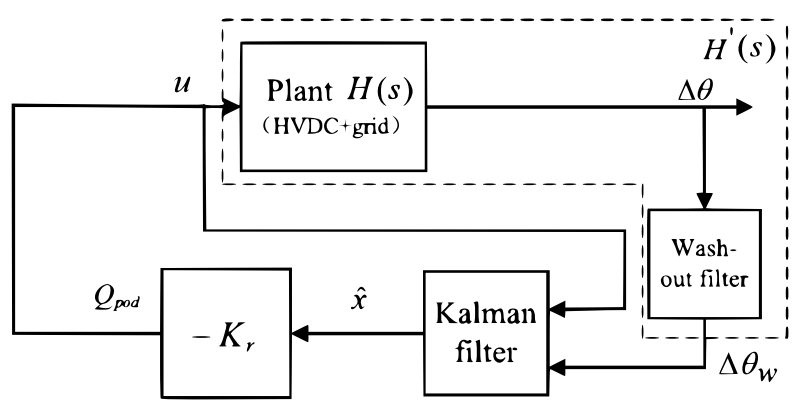

Fig. 5. The implemented control structure.

\section{B. Validation on the full linear system}

The developed LQG POD controller has been designed based on the control model $H^{\prime}(s)$ in Fig. 5 for a damping target of $10 \%$ for all modes in question. Table II reports the obtained damping results in the following situations: i) without POD, ii) with classic POD and iii) with LQG POD. 
TABLE II

COMPARISON OF DAMPING

\begin{tabular}{c|c|c|c}
\hline No.mode & $\begin{array}{c}\text { Damping without } \\
\text { POD }\end{array}$ & $\begin{array}{c}\text { Damping of modes } \\
\text { with } \\
\text { classic POD (\%) }\end{array}$ & $\begin{array}{c}\text { Damping of } \\
\text { modes } \\
\text { with LQG } \\
\text { POD }(\%)\end{array}$ \\
\hline \hline 1 & 19.5 & 30.5 & 21.7 \\
2 & 4.5 & 6.1 & 10.9 \\
3 & 10.1 & 12.0 & 14.9 \\
4 & 8.3 & 8.1 & 11.4 \\
5 & 10.1 & 12.4 & 13.6 \\
\hline
\end{tabular}

The proposed controller achieves the control requirements as the damping of all modes $(\Lambda)$ is improved and larger than the fixed target (10\%). This is due to the amount of information about the modes with close frequencies contained in the model used for the controller design. This is compared with the results obtained of the classic IEEE POD controller, which uses less information and neglects the other modes (refer to Section II.C for a detailed analysis). As a consequence, the target damping is not reached for all modes, even not for mode 2 for which the tuning is performed.

To highlight the effect of the proposed controller on the whole frequency band, we have considered an impulse response of the full linearized model of the power system in the above three situations. Fig. 6 illustrates the output of the plant $(\Delta \theta)$ under such an impulse. It can be seen that the output response with the LQG controller has clear damped oscillations compared to the classic POD and the open-loop responses. This qualitative behaviour is coherent with the modal analysis estimation in Table II. Notice also that the damping of mode 1 is lower with the LQG control than with the classic one. This is not an issue since the level is much above the target of $10 \%$ and it is normal since a globally (for all modes) optimal result is expected with the LQG control.

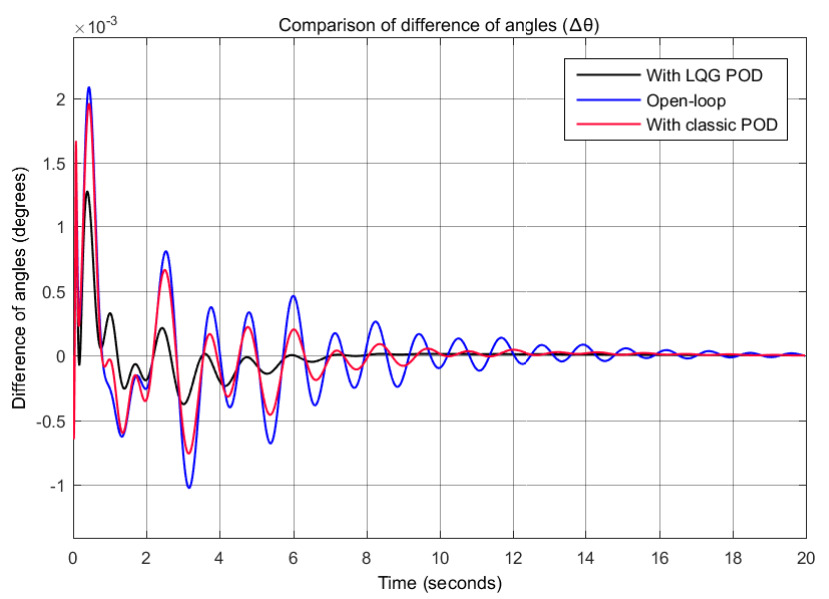

Fig. 6. Comparison of $\Delta \theta$ between open-loop system, system with classic POD controller and system with LQG POD controller in full linear model simulation under condition of an impulse signal as reference input.

\section{Validation on the nonlinear system}

In this subsection, we consider the nonlinear model of the power system with VSC-HVDC link in the same three situations studied in the previous subsection. Simulations were performed in Eurostag and plotted in Matlab.

Due to nonlinearities, the analysis is here more difficult. Each response curve captures the contribution of several modes mixed with nonlinear dynamics. It is thus difficult to put into evidence each mode individually. The disturbance scenarios should be well chosen. Also the observability of the modes depends on the choice of the output signal (generator and variable to be displayed).

To excite the lowest damped inter-area mode (mode 2), we consider a short circuit at the terminal of generator (GE_918). Fig. 7 illustrates the speed response of generator GE_911 in open-loop, with classic POD and with LQG POD controller. One can notice that mode 2 is observable from the 7 th swing in open-loop curve and its frequency can be measured from two peaks. High oscillations (close to $4.5 \%$ ) can be observed for the open-loop response. However, these oscillations are clearly damped by LQG-POD controller. Besides, the damping is lower with classic POD controller.

Consider now a short circuit at terminal of generator GE_911. Mode 4 is observable in the responses of GE_912 in Fig. 8 after 3 swings as this generator is the most participating in mode 4. It is damped with the LQG controller, but close to the open-loop value with the classic POD. Mode 1 is observable in first two swings of Fig. 9 which gives the responses of GE_914 to the same short circuit. Notice that the damping in this case is again above the one provided by the classic controller. The LQG POD controller guarantees thus a sufficient damping in a wide frequency band which contains several modes and of different nature.

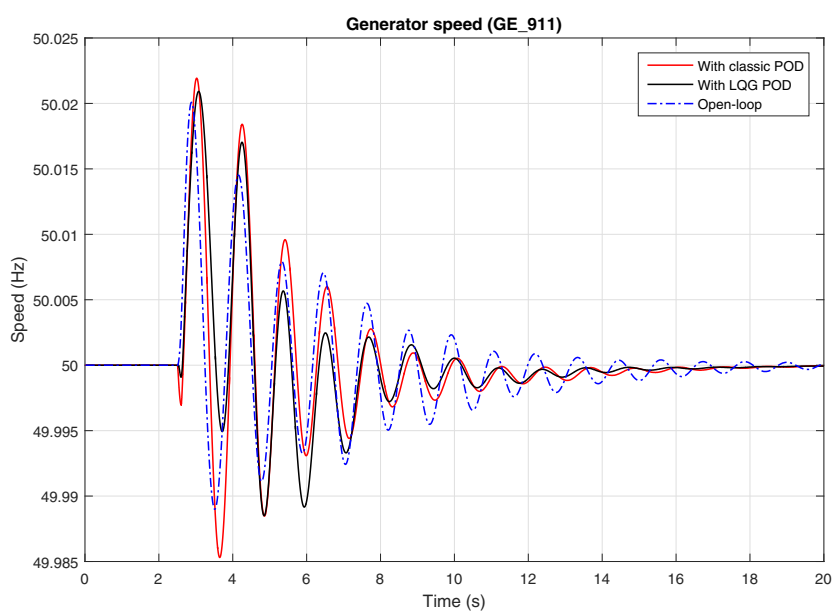

Fig. 7. Comparison of generator (GE_911) speed responses of open-loop system and system with LQG POD controller.

\section{CONCLUSIONS AND PERSPECTIVES}

The problem of power oscillations damping control of a VSC-HVDC was addressed in the particular context where the link is inserted in a meshed AC grid. The only information of the residues of the modes to be damped is not sufficient in 


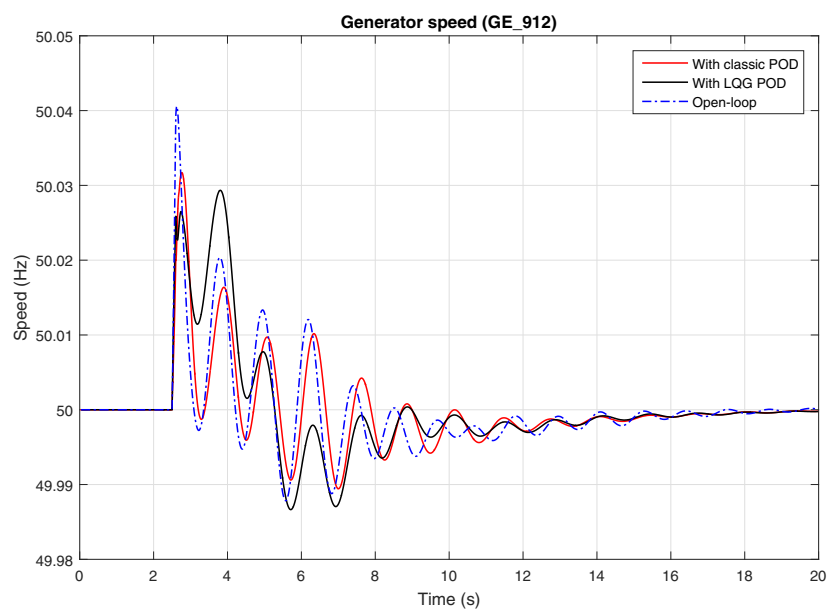

Fig. 8. Comparison of generator (GE_912) speed responses of open-loop system and system with LQG POD controller.

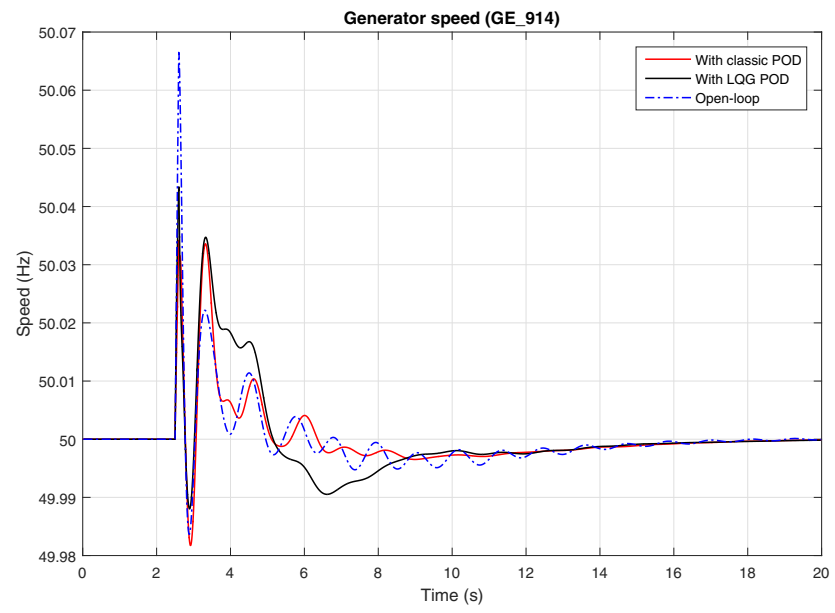

Fig. 9. Comparison of generator (GE_914) speed responses of open-loop system and system with LQG POD controller.

this case and a dynamic control model which captures not only the modes to be damped but also the other dynamics which might be excited by the closed-loop is proposed. A low order representation is obtained for this model even in case of large-scale grids. An advanced state-space robust control based on this model was given. This allowed us to provide better performances (higher damping than with the classic controller on target modes) and to avoid undesirable interactions with modes of different nature which are at near frequencies with the target inter-area modes.

Performance of the proposed LQG POD controller was proven to be superiority of the one of classic POD mainly for the nominal case. To further improve robustness against the effect of nonlinearities, change of operating point and other uncertainties in large-scale system (due to load/topology variation or faults), Loop Transfer Recovery (LTR) can be used in conjunction with LQG like in [7] or more advanced pole placement methods (like robust $H_{\infty}, H_{2}$ or fuzzy control).

The approach will be extended to active power modulation. The usage of the control model will particularly allow coordination of the three modulations (active power and reactive power at both sides of the link). Also, other measured signals will be considered according to [14].

\section{ACKNOWLEDGMENT}

The authors would like to thank Dr. Mohammed Benmiloud, post-doc at Ecole Centrale de Nantes, for his helpful suggestions and China Scholarship Council (CSC) for the financial support to Yankai XING.

\section{REFERENCES}

[1] H. F. Latorre, M. Ghandhari, and L. Söder, "Active and reactive power control of a vsc-hvdc," Electric Power Systems Research, vol. 78, no. 10, pp. 1756-1763, 2008.

[2] L. Arioua and B. Marinescu, "Robust grid-oriented control of high voltage dc links embedded in an ac transmission system," International Journal of Robust and Nonlinear Control, vol. 26, no. 9, pp. 1944-1961, 2016.

[3] — , "Multivariable control with grid objectives of an hvdc link embedded in a large-scale ac grid," International journal of electrical Power \& Energy Systems, vol. 72, pp. 99-108, 2015.

[4] "Ieee recommended practice for excitation system models for power system stability studies (ieee std 421.5-2005)," Energy Development and Power Generating Committee of the Power Engineering Society, vol. 95, p. 96, 2005.

[5] K. Vance, A. Pal, and J. S. Thorp, "A robust control technique for damping inter-area oscillations," in Power and Energy Conference at Illinois (PECI), 2012 IEEE. IEEE, 2012, pp. 1-8.

[6] A. C. Zolotas, P. Korba, B. Chaudhuri, and I. M. Jaimoukha, "H2 lmi-based robust control for damping oscillations in power systems," in System of Systems Engineering, 2007. SoSE'07. IEEE International Conference on. IEEE, 2007, pp. 1-8.

[7] R. Preece, J. V. Milanovic, A. M. Almutairi, and O. Marjanovic, "Damping of inter-area oscillations in mixed ac/dc networks using wams based supplementary controller," IEEE Transactions on Power Systems, vol. 28, no. 2, pp. 1160-1169, 2013.

[8] B. Chaudhuri and B. C. Pal, "Robust damping of multiple swing modes employing global stabilizing signals with a tcsc," IEEE Transactions on Power Systems, vol. 19, no. 1, pp. 499-506, 2004.

[9] O. Kotb, M. Ghandhari, J. Renedo, L. Rouco, and R. Eriksson, "On the design and placement of a supplementary damping controller in an embedded vsc-mtdc network," in Innovative Smart Grid Technologies Conference Europe (ISGT-Europe), 2017 IEEE PES. IEEE, 2017, pp. $1-6$.

[10] S. Skogestad and I. Postlethwaite, Multivariable feedback control: analysis and design. Wiley New York, 2007, vol. 2.

[11] B. Marinescu and D. Petesch, "Three-level coordination in power system stabilization," Electric Power Systems Research, vol. 111, pp. 40-51, 2014.

[12] B. Meyer and M. Stubbe, "Eurostag, a single tool for power system simulation," Transmission and Distribution International, vol. 3, no. 1, pp. 47-52, 1992.

[13] L. Rouco, "Coordinated design of multiple controllers for damping power system oscillations," International journal of electrical power \& energy systems, vol. 23, no. 7, pp. 517-530, 2001.

[14] M. Belhocine, B. Marinescu, and F. Xavier, "Input signal and model structure analysis for the hvdc link pod control," in PowerTech, 2017 IEEE Manchester. IEEE, 2017, pp. 1-6.

[15] G. Rogers, Power system oscillations. Springer Science \& Business Media, 2012.

[16] M. A. Hassouneh, H.-C. Lee, and E. H. Abed, "Washout filters in feedback control: Benefits, limitations and extensions," in American Control Conference, 2004. Proceedings of the 2004, vol. 5. IEEE, 2004, pp. 3950-3955.

[17] K. Zhou and J. C. Doyle, Essentials of robust control. Prentice hall Upper Saddle River, NJ, 1998, vol. 104. 